\title{
Spin-Phonon Chains with Bond Coupling
}

\author{
Carsten Raas, f Ute Löw, fil and Götz S. Uhrigf \\ Institut für Theoretische Physik, Universität zu Köln, Zülpicher Str. 77, D-50937 Köln, Germany. \\ Rainer W. Kühne \\ Institut für Theoretische Physik, Universität Dortmund, D-44221 Dortmund, Germany.
}

\begin{abstract}
We investigate the antiadiabatic limit of an antiferromagnetic $S=1 / 2$ Heisenberg chain coupled to Einstein phonons via a bond coupling. The flow equation method is used to decouple the spin and the phonon part of the Hamiltonian. In the effective spin model longer range spin-spin interactions are generated. The effective spin chain is frustrated. The resulting temperature dependent couplings are used to determine the magnetic susceptibility and to determine the phase transition from a gapless state to a dimerized gapped phase. The susceptibilities and the phase diagram obtained via the effective couplings are compared with independently calculated quantum Monte Carlo results.
\end{abstract}

PACS numbers: 63.20.Ls, 75.10.Jm, 75.50.Ee, 63.20.Kr

\section{INTRODUCTION}

We study the magnetic susceptibility and the zerotemperature phase diagram of the antiferromagnetic $S=$ $1 / 2$ Heisenberg chain coupled to dispersionless Einstein phonons. Due to the coupling of the lattice degrees of freedom to quasi one-dimensional magnetic degrees of freedom, this model exhibits a zero-temperature phase transition between a gapless phase and a massive phase showing dimerization. This quantum phase transition in the one-dimensional model is regarded as the equivalent to the spin-Peierls transitionl occurring at finite temperature in the three-dimensional model. Models of this type were investigated earlier by Pyttel and Cross and Fisher ${ }^{3}$. The discovery of the first inorganic spin-Peierls substance $\mathrm{CuGeO}_{3}$ 咱 renewed the interest in models with spin-phonon (SP) coupling. Various methods (e.g. density matrix renormalization 6 , cutoff renormalization groud, linked cluster expansions, exact diagonalizatipn 100 flow equations 11.22 , and quantum Monte Carlo 13.44.15) were used to investigate ground state properties, especially the phase diagram, the magnetic excitation spectrum and thermodynamic properties of the spin-phonon chain.

In this paper we compare results of two independent methods applied to the spin chain Hamiltonian with a local bond coupling to phononic degrees of freedom: flow equations (continuous unitary transformations) and quantum Monte Carlo. With the flow equation method we map the initial magneto-elastic problem onto an effective magnetic problem with temperature dependent effective spin-spin couplings. These are used to calculate the magnetic susceptibility (via high temperature series expansions or exact complete diagonalization) and to determine the $T=0$ phase separation line. Independently, the phase diagram and $\chi(T)$ are determined by means of quantum Monte Carlo (QMC).

In Sect. II we introduce the model Hamiltonian for the spin-phonon chain and present the flow equation approach used. Next, the quantum Monte Carlo approach is introduced in Sect. III. Finally, we show our results for the $T=0$ phase diagram in Sect. IV A and for the magnetic susceptibility in Sect. IVB. A short summary is given in Sect. V.

\section{DEFINITION OF THE MODEL AND THE FLOW EQUATIONS}

The model under study reads

$$
\begin{aligned}
\mathcal{H}(\ell) & =\mathcal{H}_{\mathrm{S}}+\mathcal{H}_{\mathrm{SB}}+\mathcal{H}_{\mathrm{B}} \\
\mathcal{H}_{\mathrm{S}} & =\sum_{i}\left(J_{1}(\ell) \mathbf{S}_{i} \mathbf{S}_{i+1}+J_{2}(\ell) \mathbf{S}_{i} \mathbf{S}_{i+2}\right) \\
\mathcal{H}_{\mathrm{B}} & =\omega \sum_{i} b_{i}^{\dagger} b_{i} \\
\mathcal{H}_{\mathrm{SB}} & =\sum_{i}\left(\mathcal{A}_{i}(\ell) b_{i}^{\dagger}+\mathcal{A}_{i}^{\dagger}(\ell) b_{i}\right) .
\end{aligned}
$$

Here $\mathbf{S}_{i}$ stands for the $S=1 / 2$ spin operator on site $i$ and $b_{i}\left(b_{i}^{\dagger}\right)$ destroys (creates) a phonon on the bond between the sites $i$ and $i+1$. The variable $\ell$ is used to parameterize the flow equation transformation and is explained in detail in the following paragraph. $\mathcal{H}_{\mathrm{S}}$ is the Hamiltonian of a frustrated spin chain or $J_{1}-J_{2}$ model. If the frustration parameter $\alpha \equiv J_{2} / J_{1}$ exceeds a critical value of $\alpha=\alpha_{\mathrm{c}}=0.241167(5)$, the model undergoes a quantum phas transition from a gapless state to a gapped phase 16.17 18. We start with the Hamilton operator (1) consisting of pure spin $\left(\mathcal{H}_{\mathrm{S}}\right)$ and phonon $\left(\mathcal{H}_{\mathrm{B}}\right)$ parts and a spin-phonon coupling term $\mathcal{H}_{\mathrm{SB}}$. Flow equations are used to decouple the spin-phonon system.

The flow equation approach was introduced by Wegner in 1994t920. This method is similar to Fröhlich's approach 21, which maps a Hamilton operator onto an effective Hamiltonian in one step by applying a unitary transformation. Wegner's approach constitutes a modification of Fröhlich's single step transformation since an infinite number of infinitesimal unitary transformations 
is used. The idea behind this scheme is that the continued adjustment of the infinitesimal transformation to the Hamiltonian yields a smoother effective interaction than a rotation in one step.

The continuous unitary transformation is parameterized by a flow parameter $\ell \in[0, \infty]$. So $\mathcal{H}(\ell=0)$ stands for the original Hamiltonian. The effective Hamiltonian $\mathcal{H}(\ell=\infty)$ is simpler in the way that the direct spinphonon coupling has been rotated away. The infinitesimal generator $\eta(\ell)$ defines the unitary transformation via

$$
\frac{\mathrm{d} \mathcal{H}(\ell)}{\mathrm{d} \ell}=[\eta(\ell), \mathcal{H}(\ell)] .
$$

A choice of $\eta(\ell)$ proposed by Wegner is

$$
\eta(\ell)=\left[\mathcal{H}_{\mathrm{d}}(\ell), \mathcal{H}(\ell)\right],
$$

where $\mathcal{H}_{\mathrm{d}}$ is the part of the Hamiltonian which is taken as the diagonal part. By choosing $\mathcal{H}_{\mathrm{d}}(\ell) \equiv \mathcal{H}_{\mathrm{S}}(\ell)+\mathcal{H}_{\mathrm{B}}(\ell)$ and the "off-diagonal" interaction part as $\mathcal{H}_{\text {od }}(\ell) \equiv$ $\mathcal{H}_{\mathrm{SB}}(\ell)$, our generator reads $\eta(\ell) \equiv\left[\mathcal{H}_{\mathrm{d}}, \mathcal{H}_{\mathrm{od}}\right]=\left[\mathcal{H}_{\mathrm{S}}+\right.$

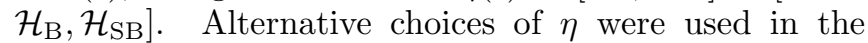
literature22.2324 in order to obtain flow equations with specific properties. For the present purposes, the canonical choice (3) of $\eta$ is sufficient.

As initial conditions we choose

$$
J_{1}(0)=J \quad \text { and } \quad J_{2}(0)=J_{2}^{0}=0 .
$$

So the untransformed pure spin part is the antiferromagnetic $S=1 / 2$ Heisenberg Hamiltonian with a nearest neighbor coupling. In the limit $\ell \rightarrow \infty$ longer range spin-spin interactions arise and the next-nearest neighbor coupling constant $J_{2}$ in the effective spin model becomes finite.

$\mathcal{H}_{\mathrm{SB}}$ describes the coupling between spin and phonon system. The coupling operator $\mathcal{A}_{i}(0)$ typically consists of nearest neighbor spin products since in realistic magnetic materials the spin-phonon coupling mainly influences neighboring sites. By choosing different coupling operators various mechanisms of how the lattice distortions influence the exchange integral $J$ can be investigated. If the exchange coupling depends on the position of a spin between the neighboring ones the appropriate choice for $\mathcal{A}_{i}(0)$ is the difference coupling

$$
\mathcal{A}_{i}^{\text {diff }}=g\left(\mathbf{S}_{i} \mathbf{S}_{i+1}-\mathbf{S}_{i} \mathbf{S}_{i-1}\right) .
$$

For $\mathrm{CuGeO}_{3}$ this corresponds to changes of two neighboring $\mathrm{Cu}-\mathrm{O}-\mathrm{Cu}$ binding angles induced by shifts of the copper ion in the middle, i. e. one angle is enlarged at the expense of the other one. This coupling type was investigated in the framework of a flow equation approach in Refs. 11 12. In Ref. 11 an analysis is presented in leading order in $g / \omega$ and in the two leading orders in $J / \omega$. Though only systematic to order $g^{2} / \omega^{2}$ and $g^{2} J / \omega^{3}$ a good qualitative picture for the antiadiabatic limit of the spin-phonon chain could be achieved. A more subtle analysis is given in Ref. 12. The price paid in Ref.
12 for high precision results for small $J / \omega$ and $g / \omega$ is a breakdown of the formalism for large values of $J / \omega$.

In this paper we study the bond coupling

$$
\mathcal{A}_{i}(0)=g \mathbf{S}_{i} \mathbf{S}_{i+1},
$$

which is frequently used $1013.15,25$, especially in connection with $\mathrm{CuGeO}_{3}$. For this type of coupling single harmonic degrees of freedom directly modify the magnetic interaction. This is due to side group effects engendered by the germanium atoms 26-2728. In the present paper we restrict ourselves to an analysis in the leading orders as in Ref. 11.

To study the Hamiltonian (11) with the coupling operator (6) via the flow equation method, let us first observe that according to Ref. 19, $\mathcal{A}_{i}$ should be normal-ordered, i. e. $\mathcal{A}_{i}(0) \rightarrow \mathcal{A}_{i}(0)-\left\langle\mathcal{A}_{i}(0)\right\rangle$. In this way the quantum phonons only couple to the comparably small spin fluctuations and not to the bare operator $\mathbf{S}_{i} \mathbf{S}_{i+1}$. This is an extremely important point. Only the coupling to the small fluctuations justifies the expansion approach. This is not guaranteed by the specific choice (6). This feature of the coupling type (6) is in contrast to the difference coupling $\mathcal{A}_{i}^{\text {diff }}$ which does not contribute to the undistorted phase due to the translational invariance 11 , i. e. $\left\langle\mathcal{A}_{i}^{\text {diff }}(0)\right\rangle$ vanishes. The necessary normal-ordered coupling operator for the bond coupling reads

$$
\mathcal{A}_{i}(0)=g\left(\mathbf{S}_{i} \mathbf{S}_{i+1}-C\right)
$$

where $C$ stands for the (temperature dependent) expectation value of the nearest neighbor spin product $\left\langle\mathbf{S}_{i} \mathbf{S}_{i+1}\right\rangle$. The value of $C$ is calculated using Padé approximants for the series expansions given in Refs. 24,29. As we determine $C$ in the uniform phase it can be interpreted as an average value over the chain For the antiferromagnetic chain the expectation value $C$ for $T=0$ lies between $1 / 4-\ln (2) \approx-0.443$ (for $\alpha=0$ ) and $3 / 8=-0.375$ (for the fully dimerized chain).

To achieve normal-ordering we can rewrite the Hamiltonian (11) by shifting the phonons operators via $b_{i} \rightarrow$ $\hat{b}_{i}-\frac{g C}{\omega}$. Thus, the Hamiltonian with the normal-ordered coupling is

$$
\begin{aligned}
\hat{\mathcal{H}}= & \hat{J} \sum_{i} \mathbf{S}_{i} \mathbf{S}_{i+1}+g \sum_{i}\left(\mathbf{S}_{i} \mathbf{S}_{i+1}-C\right)\left(\hat{b}_{i}^{\dagger}+\hat{b}_{i}\right) \\
+ & \omega \sum_{i} \hat{b}_{i}^{\dagger} \hat{b}_{i}+\sum_{i} \hat{E}_{0} \\
& \hat{J}=J-\frac{2 g^{2} C}{\omega} \quad \text { and } \quad \hat{E}_{0}=\frac{g^{2} C^{2}}{\omega} .
\end{aligned}
$$

We now follow the outline of Ref. 11. Applying the unitary transformation generated by $\eta$, we find an effective 
Hamilton operator without spin-phonon coupling terms

$$
\begin{aligned}
\mathcal{H}_{\mathrm{eff}}= & \hat{J} \sum_{i} \mathbf{S}_{i} \mathbf{S}_{i+1}+\Delta \mathcal{H}_{\mathrm{X}}+\Delta \mathcal{H}_{\mathrm{Y}} \\
& +\omega \sum_{i} \hat{b}_{i}^{\dagger} \hat{b}_{i}+\sum_{i} \hat{E}_{0} \\
\Delta \mathcal{H}_{\mathrm{X}}= & -\frac{1}{\omega} \sum_{i} \mathcal{A}_{i}^{\dagger} \mathcal{A}_{i} \\
= & \frac{g^{2}}{2 \omega} \sum_{i}\left[(1+4 C) \mathbf{S}_{i} \mathbf{S}_{i+1}-2 C^{2}-3 / 8\right] \\
\Delta \mathcal{H}_{\mathrm{Y}}= & \frac{1}{2 \omega^{2}} \operatorname{coth}\left(\frac{\omega}{2 T}\right) \sum_{i}\left[\mathcal{A}_{i},\left[\mathcal{H}_{\mathrm{S}}, \mathcal{A}_{i}\right]\right] \\
= & \frac{g^{2}}{2 \omega^{2}} \operatorname{coth}\left(\frac{\omega}{2 T}\right) \sum_{i}\left[\left(J_{2}^{0}-\hat{J}\right) \mathbf{S}_{i} \mathbf{S}_{i+1}\right. \\
& \left.+\left(\hat{J}-2 J_{2}^{0}\right) \mathbf{S}_{i} \mathbf{S}_{i+2}+J_{2}^{0} \mathbf{S}_{i} \mathbf{S}_{i+3}\right] .
\end{aligned}
$$

As no frustration is present in the original model (i. e. $J_{2}^{0}=0$ ) we obtain for the effective nearest and nextnearest neighbor couplings

$$
\begin{array}{r}
J_{1}=J+\frac{g^{2}}{2 \omega}-\frac{\hat{J} g^{2}}{2 \omega^{2}} \operatorname{coth}\left(\frac{\omega}{2 T}\right) \\
\text { and } J_{2}=\frac{\hat{J} g^{2}}{2 \omega^{2}} \operatorname{coth}\left(\frac{\omega}{2 T}\right)
\end{array}
$$

while longer range two-spin couplings and products with four different spins are omitted. In this way the dressing of the spins with phonons induces a frustration $\alpha_{\text {eff }}=$ $J_{2} / J_{1}>0$. The omission of the longer range and the four-spin couplings contributes an additional approximation. It is justified by the fact that these terms contribute neither in a Néel state nor in a dimer state. We note that the couplings are temperature dependent via the coth terms and via the $T$-dependence of the expectation value $C=\left\langle\mathbf{S}_{i} \mathbf{S}_{i+1}\right\rangle$. Though only systematic to order $g^{2} / \omega^{2}$ and $g^{2} J / \omega^{3}$, higher order effects enter due to the appearance of $\hat{J}=J-\frac{2 g^{2} C}{\omega}$ in Eqs. (10). Since $C$ is calculated self-consistently, this is in principle a correction of infinite order. In this way the phonon-shift $b_{i} \rightarrow \hat{b}_{i}-\frac{g C}{\omega}$, which is necessary to apply the flow equation approach, induces infinite order contributions.

It was pointed out in recent articles 1144,30 that the spin-Peierls transition in the antiadiabatic limit is not accompanied by a softening of the Peierls-active phonon modes as it is the case for organic spin-Peierls compounds. This phonon hardening was observed for the difference coupling using a similar flow equation approach in Ref. 12. For the bond coupling investigated here a simple formula for the effective phonon frequency $\omega_{\text {eff }}$ can be derived by evaluating the $b_{\vec{q}}^{\dagger} b_{\vec{k}}$ term of $\mathrm{d} \Delta \mathcal{H} / \mathrm{d} \ell$ (cf. formula (9) of Ref. 11). Hence we find

$$
\omega_{\text {eff }}=\omega+\frac{g^{2} \hat{J}}{\omega^{2}}\left(-\left\langle\mathbf{S}_{i} \mathbf{S}_{i+1}\right\rangle+\left\langle\mathbf{S}_{i} \mathbf{S}_{i+2}\right\rangle\right)
$$

where non-local terms are neglected. As for zero temperature $\left\langle\mathbf{S}_{i} \mathbf{S}_{i+1}\right\rangle<0,\left\langle\mathbf{S}_{i} \mathbf{S}_{i+2}\right\rangle>0$, and thus $\hat{J}=$

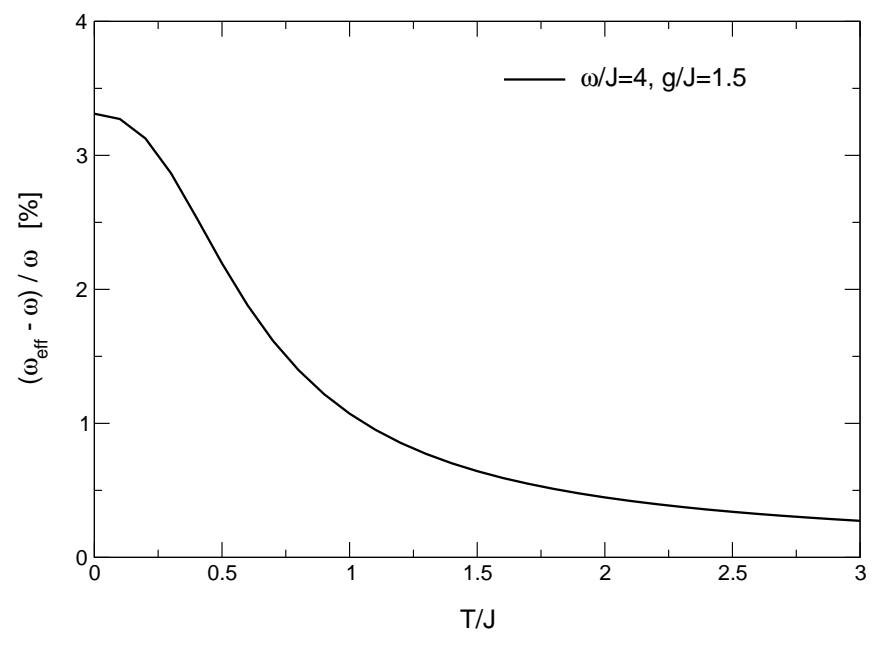

FIG. 1: Temperature dependence of the effective phonon frequency. The plot shows the relative change in $\omega$ for $\omega / J=4$ and $g / J=1.5$.

$J-2 g^{2}\left\langle\mathbf{S}_{i} \mathbf{S}_{i+1}\right\rangle / \omega>J$ the effective phonon frequency is enlarged. With increasing temperature the correlations are destroyed and accordingly $\omega_{\text {eff }}$ tends to $\omega$. The generic temperature dependence of the relative frequency increase is depicted in Fig. 1 for $\omega / J=4$ and $g / J=1.5$.

After these remarks regarding the phonon part of the effective Hamiltonian, we now turn back to the physics in the spin sector. By solving $\alpha=J_{2} / J_{1}=\alpha_{\mathrm{c}}$ for $\mathrm{g} / \mathrm{\omega}$ for $T=0$ we obtain an explicit formula for the phase separation line

$$
\frac{g_{\mathrm{c}}^{2}}{\omega^{2}}=\frac{\gamma_{\mathrm{c}}-\sqrt{\left(\gamma_{\mathrm{c}}-\alpha_{\mathrm{c}}\right)^{2}-16 C \alpha_{\mathrm{c}} \gamma_{\mathrm{c}}}-\alpha_{\mathrm{c}}}{4 C\left(1+\alpha_{\mathrm{c}}\right)}
$$

with the abbreviation $\gamma_{\mathrm{c}}=\left(1+\alpha_{\mathrm{c}}\right) J / \omega$. The two limiting cases are then given by

$$
\begin{aligned}
& \lim _{J / \omega \rightarrow 0} \frac{g_{\mathrm{c}}}{\omega}=\sqrt{\frac{-\alpha_{\mathrm{c}}}{2 C\left(1+\alpha_{\mathrm{c}}\right)}} \approx 0.4682 \\
& \lim _{J / \omega \rightarrow \infty} \frac{g_{\mathrm{c}}}{\omega}=\sqrt{\frac{2 \alpha_{\mathrm{c}}}{1+\alpha_{\mathrm{c}}}} \approx 0.6234
\end{aligned}
$$

The finiteness of the critical spin-phonon coupling $g_{\mathrm{c}} / \omega$ for $J / \omega \rightarrow 0$ is a striking difference between the local coupling mechanism considered here and the coupling to the order parameter used e.g. in Ref. 12. A qualitative understanding can be achieved by considering the shifted and rescaled Hamiltonian

$$
\begin{aligned}
\frac{\mathcal{H}}{\omega}= & {\left[\frac{J}{\omega}-2\left(\frac{g}{\omega}\right)^{2} C\right] \sum_{i} \mathbf{S}_{i} \mathbf{S}_{i+1}+\sum_{i} \hat{b}_{i}^{\dagger} \hat{b}_{i} } \\
& +\sum_{i} \frac{\hat{E}_{0}}{\omega}+\frac{g}{\omega} \sum_{i}\left(\mathbf{S}_{i} \mathbf{S}_{i+1}-C\right)\left(\hat{b}_{i}^{\dagger}+\hat{b}_{i}\right) .
\end{aligned}
$$

If $g / \omega$ is finite the effective nearest neighbor coupling constant remains finite even in the limit $J / \omega \rightarrow 0$, as the 


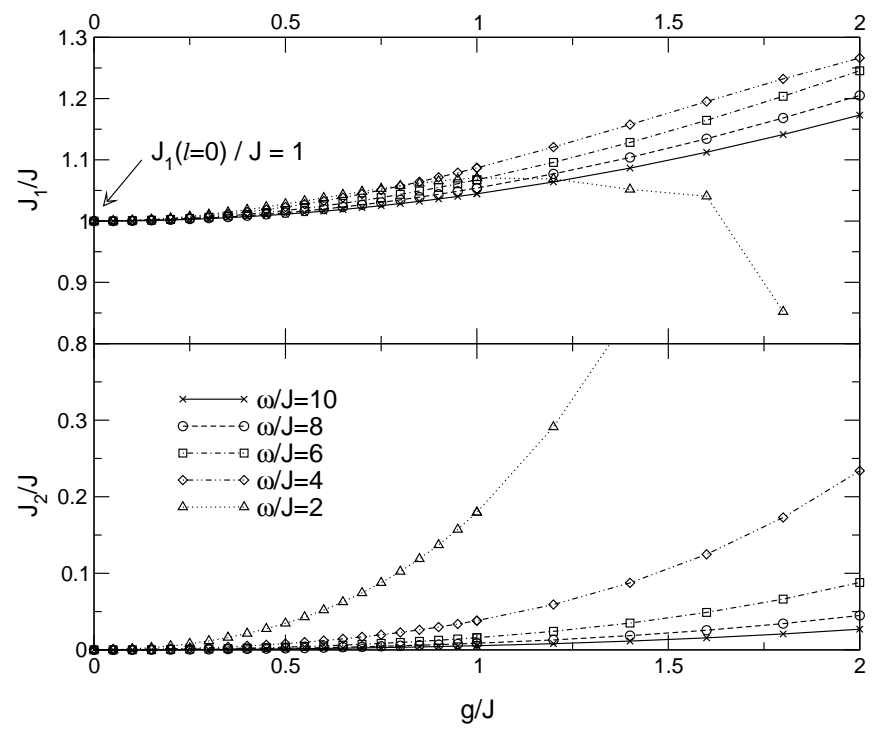

FIG. 2: Dependence of the effective nearest and next-nearest neighbor spin-spin couplings from the spin-phonon coupling $g$ for fixed values of $\omega$ and zero temperature.

expectation value $C$ is typically $\approx-0.4$. Thus, there is no reason why the critical spin-phonon coupling should vanish for $J / \omega$ approaching zero. This illustrates an important difference between the difference coupling (5) and the bond coupling (6).

Fig. 2 shows the change of the effective spin-spin couplings with increasing spin-phonon coupling. Since our method corresponds to the leading orders of an expansion in $J / \omega$ and $g / \omega$, the approach breaks down if the ratio $J / \omega$ becomes too large. This can be seen in the unsystematic downturn of the $J_{1}(g)$ curve for $\omega=2 J$.

To obtain the magnetic susceptibilities for the spinphonon chain we follow various routes. Using the effective couplings $(10)$ we perform an exact complete diagonalization for the frustrated spin chain or use alternatively high temperature expansions 29 . In order to extrapolate the results we use Dlog-Padé approximants 3132. If the spin-phonon coupling $g / \omega$ is small, the effective frustration is negligible. This justifies the third approach to simply rescale the exact results for the unfrustrated Heisenberg chain $33.34,35$ by the renormalized nearest-neighbor coupling $J_{1}$. The results for these three methods agree perfectly well with the direct QMC data, which are not based on the derived effective couplings.

\section{QUANTUM MONTE CARLO}

In a completely independent approach we study the model (1) directly by a quantum Monte Carlo method based on the loop algorithm 36 . The technical details of the method are described in Ref. 15. Here we just point out again, that the algorithm allows to incorporate as many phonons as desired. We usually choose 50 per site.
Thus our results are not hampered by too low cutoffs in the phonon number, which represent otherwise a serious difficulty at low phonon frequencies. As has been demonstrated in Ref. 15 the error originating from the finiteness of the Trotter number can be easily overcome by extrapolation. Here Trotter numbers of $M=40,60$ and 80 are used. The calculations are performed with $10^{5}-10^{6}$ spin updates and 20 phonon updates per spin update. We considered up to 256 sites so that the finite-size effects are controllably small. Thus, apart from statistical fluctuations the Monte Carlo results are exact.

It was appropriate for the quantum Monte Garlo study to use the Hamiltonian in the following form 15

$$
\tilde{\mathcal{H}}=2 \tilde{J} \sum_{i}\left(\mathbf{S}_{i} \mathbf{S}_{i+1}-\frac{1}{4}\right)\left[1+\tilde{g}\left(b_{i}^{\dagger}+b_{i}\right)\right]+\tilde{\omega} \sum_{i} b_{i}^{\dagger} b_{i}
$$

where we use the tilde to distinguish clearly between the different coupling constants in the different ways to denote the Hamiltonian. The operator (15) can be mapped onto (1) by a shift in the phonon operators

$$
b_{i} \rightarrow b_{i}+\frac{\tilde{J} \tilde{g}}{2 \tilde{\omega}}
$$

By this mapping one obtains

$$
\begin{aligned}
\tilde{\mathcal{H}}= & 2 \tilde{J}\left(1+\frac{\tilde{J} \tilde{g}^{2}}{\tilde{\omega}}\right) \sum_{i} \mathbf{S}_{i} \mathbf{S}_{i+1}+\tilde{\omega} \sum_{i} b_{i}^{\dagger} b_{i}+ \\
& 2 \tilde{g} \tilde{J} \sum_{i} \mathbf{S}_{i} \mathbf{S}_{i+1}\left(b_{i}^{\dagger}+b_{i}\right)-\frac{\tilde{J}}{2}\left(1-\frac{\tilde{J} \tilde{g}^{2}}{2 \tilde{\omega}}\right) .
\end{aligned}
$$

Accounting for this rescaling of the spin-spin coupling constant the susceptibilities computed for $\mathcal{H}$ in Eq. (15) and those for $\mathcal{H}$ in Eq. (1) can be compared.

The quantum Monte Carlo procedure is per construction a method giving results at finite temperature $T$. But since the algorithm allows to approach very low $T$ one can also study properties of the ground state, in particular if one considers the model in its gapped phase. This was used in the following to extract the phase boundaries of the model.

\section{RESULTS}

\section{A. Phase diagram}

The calculation via flow equations is based on the idea that the transition into the ordered phase does not occur due to the softening of a phonon but due to the tendency of the effective spin model towards dimerization. Phonon-induced frustration above its critical value $\alpha_{\text {eff }}>\alpha_{\mathrm{c}}$ drives the dimerization. The phase transition line is therefore determined by solving

$$
\alpha_{\mathrm{c}}=\left.\left(\lim _{\ell \rightarrow \infty} \alpha(g)\right)\right|_{J}
$$




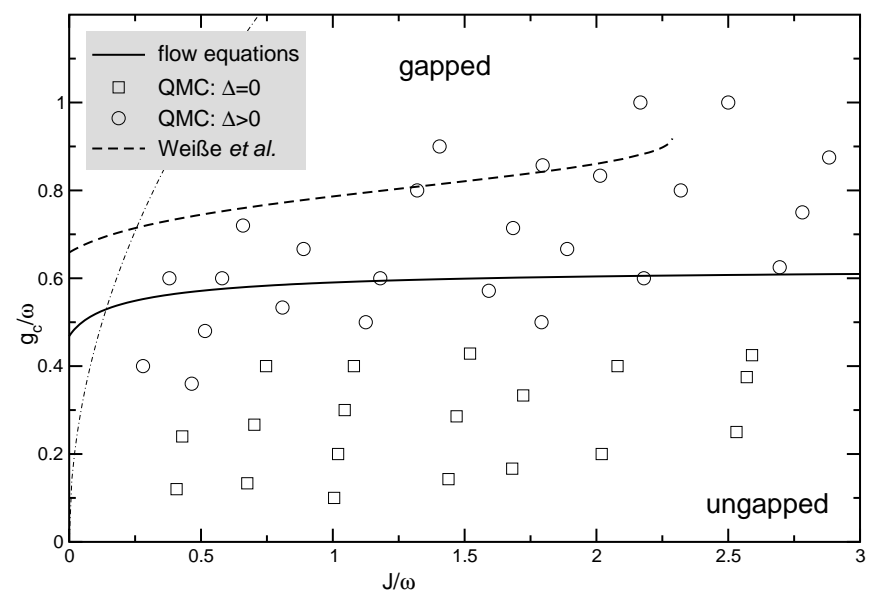

FIG. 3: Zero temperature phase diagram of the spin-Peierls antiferromagnetic chain of spins interacting with phonons. For small values of the spin-phonon coupling $g / \omega$ the system is gapless $(\Delta=0)$. For large $g / \omega$ the system is dimerized and has an energy gap $(\Delta>0)$. The circles (squares) correspond to QMC data, where (no) dimerization was found for $T / J=0.05$ and a chain of $N=256$ sites. QMC data can only be computed to the right of the dot-dashed line (for details see main text). The results from Weiße et. al. are taken from Ref. 10.

for fixed values of $J$ with respect to $g$ (cf. Eq. 12). In this way we find the critical spin-phonon coupling $g_{\mathrm{c}}$ in dependence of the nearest neighbor spin coupling $J$ as depicted in Fig. 3 .

Using the quantum Monte Carlo method the decision whether a pair $(J / \omega, g / \omega)$ represents a point in the ungapped or the gapped phase is reached by investigating the expectation values of the local phonon displacements $\left\langle b_{i}^{\dagger}+b_{i}\right\rangle$. At low temperatures $\left\langle b_{i}^{\dagger}+b_{i}\right\rangle$ show clear dimerization patterns if the model is in the gapped phase and they are randomly fluctuating in the critical phase of the model (see Fig. (1). Of course, the ergodicity of the Monte Carlo procedure complicates the identification of the ground state in the gapped phase since for long run times the ground state fluctuates always between the two possible dimerized patterns. However, by averaging over not too many configurations (we generally take 10000) one can extract well the dimerization patterns from the Monte Carlo results.

For intermediate values of $J / \omega$ the flow equation and QMC method predict similar values for the strength of the critical coupling $g_{\mathrm{c}} / \omega$ (cf. Fig. 3). There is a certain tendency that the QMC data indicates lower values for $g_{\mathrm{c}} / \omega$ than the analytical calculation. We attribute this discrepancy partly to the approximate character of the analytical calculation and partly to the difficulty to determine the phase boundary by QMC precisely. Note that due to the phonon shift (16) the QMC approach is restricted to the region on the right hand side of the dotdashed line in Fig. 3. The QMC data indicate a smaller value for $g_{\mathrm{c}} / \omega$ in the antiadiabatic limit. In the immedi-

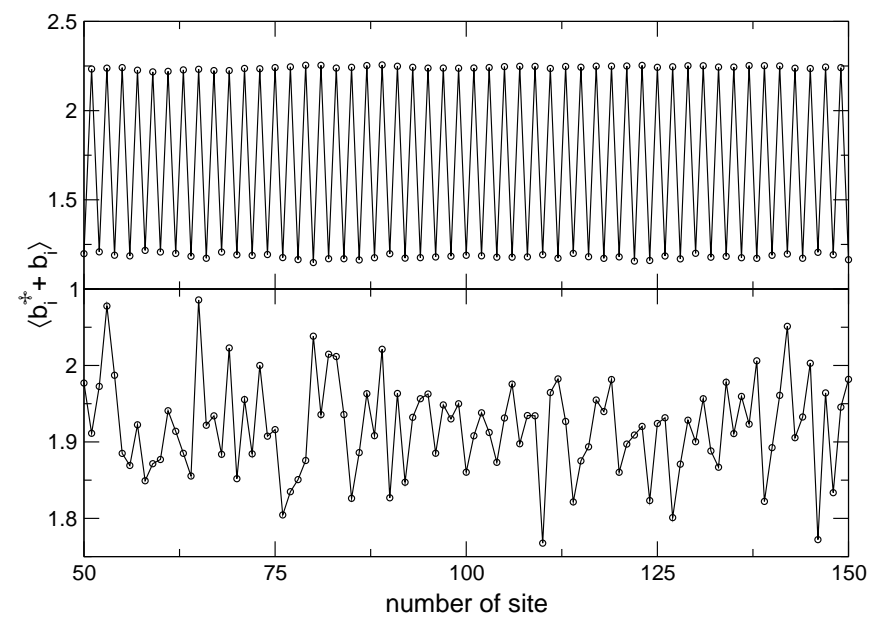

FIG. 4: Phonon displacement averaged over $10^{4}$ configurations for $\tilde{\omega}=10 J$ and $\tilde{g}=0.8(\tilde{g}=0.1)$ for a system size of $N=256$ sites in the upper (lower) plot. For $\tilde{g}=0.8$ the dimerization is clearly visible.

ate vicinity of the dot-dashed line (i. e. for very large $\tilde{\omega}$ ) it is rather hard to distinguish between a fully dimerized pattern and partially dimerized structures. Thus, further investigations are desirable for the extreme antiadiabatic limit of the phase diagram.

The phase separation line predicted by Weiße et. al. via fourth order perturbation theory lies well above the flow equation curve and above the Monte Carlo results. This was also observed in Ref. 12 where in the case of the difference coupling the leading order result 11 compared much better with the DMRG datab than the fourth order result $\Perp$. Since with a difference coupling the critical spin-phonon coupling $g_{\mathrm{c}} / \omega$ vanishes as $J / \omega$ goes to zero the expansion in these parameters becomes exact. Thus, the phase separation lines for the difference coupling coincide for small values of $J / \omega .12$ But as discussed above, in the case of a bond coupling $g_{\mathrm{c}} / \omega$ is non-zero in the antiadiabatic limit $(J / \omega \rightarrow 0)$. Hence, it is not surprising that even in the limit of vanishing $J / \omega$ the deviation does not decrease. Ref. 10 predicts even a value of $g_{\mathrm{c}} / \omega=\sqrt{8 / 3 \cdot \alpha_{\mathrm{c}} /\left(1+2 \alpha_{\mathrm{c}}\right)} \approx 0.6587$ for $J / \omega \rightarrow 0$.

In Ref. 14 a QMC analysis of the spin-phonon chain with a bond coupling was presented for the parameter $J / \omega=4$. A critical coupling $g_{\mathrm{c}} / \omega=0.636 \pm 0.042$ was calculated by investigating the staggered spin susceptibility for various system sizes. This result is in very good agreement with $g_{\mathrm{c}} / \omega=0.613$ predicted by the flow equation method (not depicted in the phase diagram Fig. 3).

\section{B. Magnetic susceptibility}

In this Section we consider the influence of the spinphonon coupling on a thermodynamic quantity like the magnetic susceptibility $\chi(T)$. We show that the magnetic 


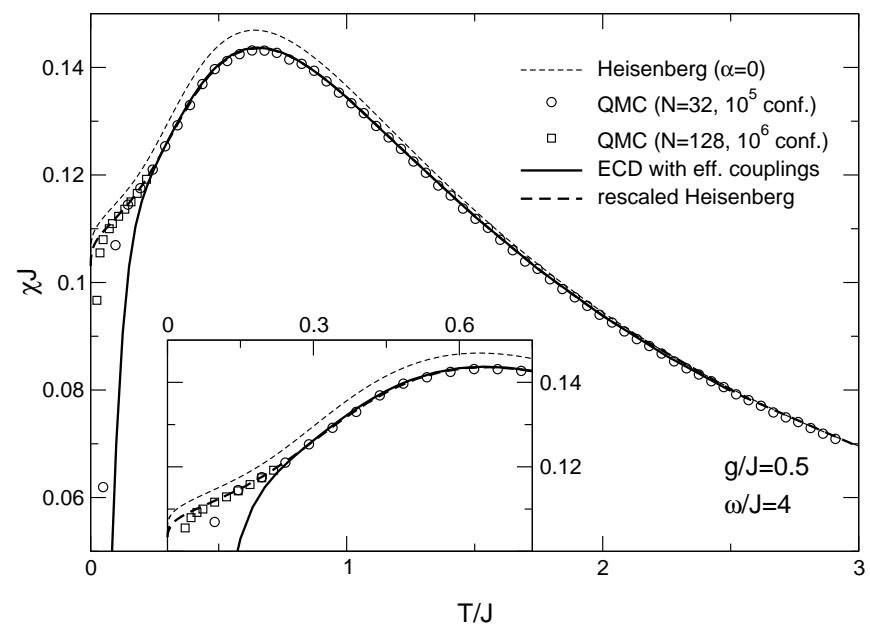

FIG. 5: Magnetic susceptibility for $g=0.5 J$ and $\omega=4 J$.

susceptibility can be well described by an effective spin model. Furthermore, the question whether it is possible to describe thermodynamic quantities of the SP chains with a static spin model or via a spin model with temperature dependent couplings $J(T)$ is investigated. The exchange integral $J$ of a quantum spin system is often assumed to be constant. However, in real materials where $J$ depends on the actual positions of the magnetic ions in the crystal this approximation need not be valid. For the spin-Peierls substance $\mathrm{CuGeO}_{3}$ this point is of special interest. The magnetic susceptibility of $\mathrm{CuGeO}_{3}$ in the temperature range above the spin-Peierls transition san be well fitted by a frustrated Heisenberg model.37.38) 39 It is often objected, however, that the agreement might be accidental as no interchain interactions and spin-phonon couplings are taken into account. This motivates us although the Hamiltonian introduced in Eq. (11) is too simple to be a realistic model for $\mathrm{CuGeO}_{3}$ - to study in detail the influence of the spin-phonon coupling on the thermodynamic observables. Another substance with strong magneto-elastic couplings, $(\mathrm{VO})_{2} \mathrm{P}_{2} \mathrm{O}_{7}$, was investigated recently 31 by a flow equation approach. The authors found that even for strong spin-phonon coupling the influence of phonons on thermodynamic magnetic quantities is rather small. A good description of such quantities could already be achieved by a static model.

The flow equation approach maps the spin-phonon chain onto a frustrated spin chain with temperature dependent spin-spin couplings. The corresponding $\chi(T)$ is compared with the susceptibility calculated with QMC for the full Hamiltonian (15). This enables us to study the influence of the spin-phonon coupling on $\chi(T)$. Furthermore, we can discuss the question whether it is possible to retrieve the main physical properties of a spinphonon chain by looking at its effective spin model.

Figures 5 -8 show our results for four different values of $g$ and $\omega$. All parameters are given according to the Hamiltonian (11). As a reference the exact result of the

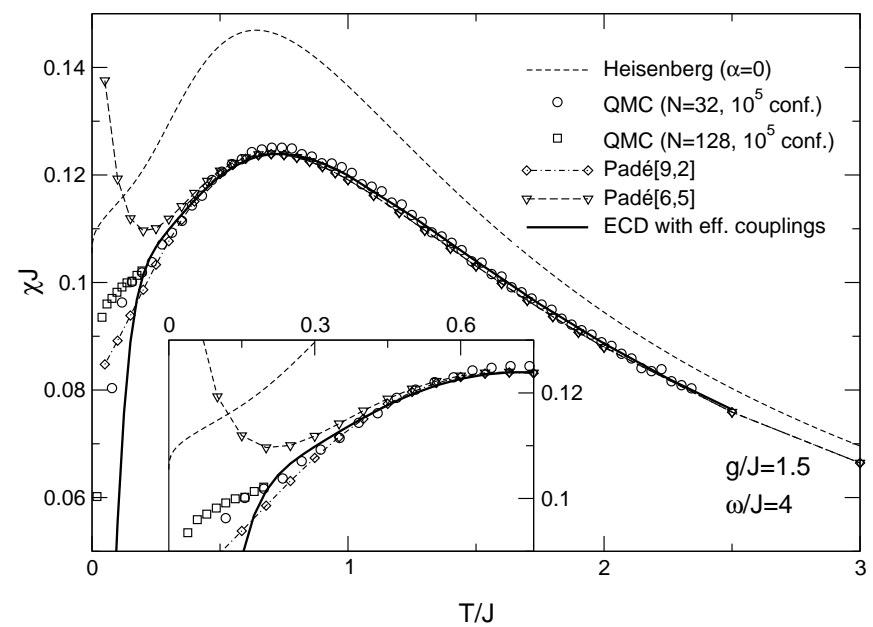

FIG. 6: Magnetic susceptibility for $g=1.5 J$ and $\omega=4 J$.

unfrustrated Heisenberg chain 33.3435 is depicted in all figures. Fig. 5 illustrates that a rather small spin-phonon coupling $g=0.5 \mathrm{~J}$ leads only to a lowering of the overall height of the susceptibility. The frustration in the effective model is very small $\left(J_{2} / J_{1} \approx 0.008\right.$ for $T=0$ and $J_{2} / J_{1} \approx 0.02$ for $\left.T / J=5\right)$. This enables us to rescale the Heisenberg curve with respect to the enlarged coupling constant $J_{1}$. To ensure that the effective frustration is really small enough to be neglected we make an additional check via the exact and complete diagonalization (ECD) data (computed with the temperature dependent couplings $J_{1}$ and $J_{2}$ ) for a frustrated chain with $N=16$ sites. The QMC and the flow equation results agree perfectly down to very low temperatures.

When $g$ is increased the maximum decreases as can be seen in Fig. 6 for $\omega=4 J$ and $g=1.5 J$. Again, the two approaches yield the same results down to $T / J \approx 0.2$. The frustration is no longer negligible. As one can see, the use of an effective spin model to describe $\chi(T)$ for a spin chain coupled to phonons is well justified. An easy way to fit experimental data is to use Padé approximants for high temperature series expansion instead of exact complete diagonalization. The maximum is welldescribed by this procedure, even though the limit of very low temperatures is problematic.

Figures 7 and 8 show additional results for $g=1.0 \mathrm{~J}$ and $g=1.5 \mathrm{~J}$ and for $\omega=10 J$. As $J / \omega$ is small the flow equation approach works especially well and thus the agreement is even better compared with the $\omega=4 J$ results.

Three final remarks regarding the susceptibility results are in order. In Ref. 15, the authors observed that with increasing spin-phonon coupling the susceptibility curves are shifted to higher temperatures. The main reason for this is the static term $\propto\left\langle b_{i}^{\dagger}+b_{i}\right\rangle$ in the original QMC Hamiltonian (15). This is not visible in our way of depicting $\chi(T)$ using the parameterization (1i) as we shifted the phonons to make this term vanish. If this static effect 


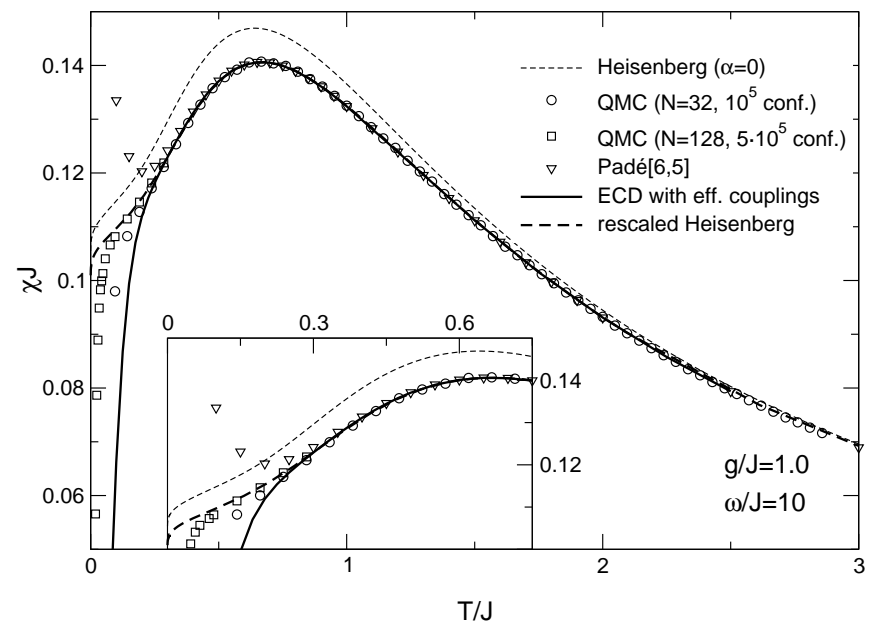

FIG. 7: Magnetic susceptibility for $g=1.0 \mathrm{~J}$ and $\omega=10 \mathrm{~J}$.

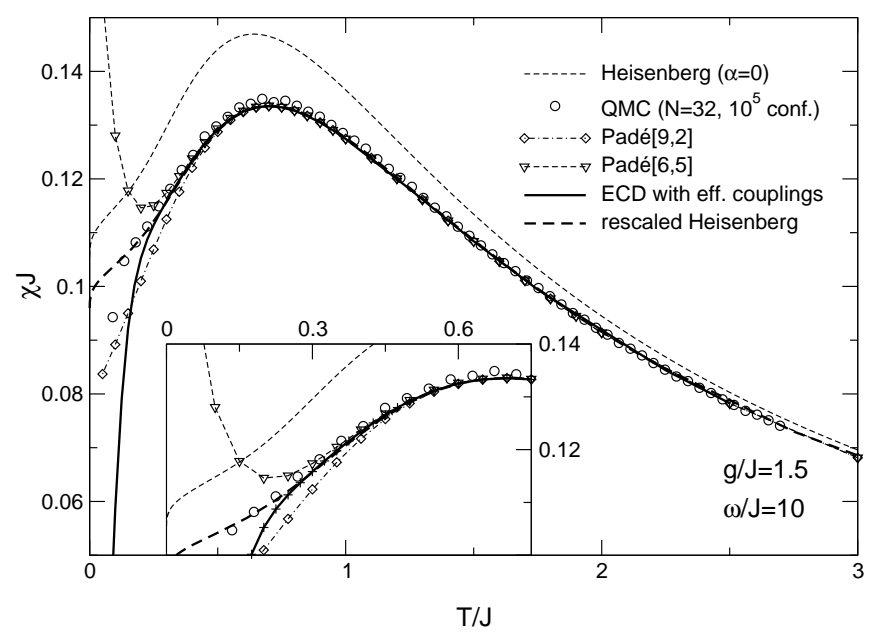

FIG. 8: Magnetic susceptibility for $g=1.5 \mathrm{~J}$ and $\omega=10 \mathrm{~J}$.

is accounted for by the phonon shift, the positions of the maxima of $\chi(T)$ are nearly the same for all parameters $g$ and $\omega$ presented here. Only if $g / \omega$ and thus the effective frustration are very large a small shift due to the temperature dependent frustration becomes visible.

The second remark concerns the applicability of the flow equation procedure presented here. As we have already seen in Fig. 2, the ratio $J / \omega$ must not become too large. Thus, we are only able to show susceptibilities with $J / \omega \lesssim 1 / 4$. The main problem for a thermodynamic quantity is the $\operatorname{coth}(\omega / 2 T)$ term in the formula (10) for the effective couplings because it diverges for $T \rightarrow \infty$. To reach higher values of $J / \omega$ a more subtle flow equation approach would be necessary. But even the straightforward flow equation results agree well with the QMC data for not too large $J / \omega$ and $g / \omega$.

The third remark concerns the temperature dependence of the effective couplings. This dependence is fairly small for the parameter values in Figs. 5 \& which is due to the small values $J / \omega$. Thus good fits are also obtained neglecting the temperature dependence $J(T) \equiv J$ in a model of static effective couplings. This is in agreement with previous results 31 . But we expect that for larger values of $J / \omega$ the temperature dependence will be important. Yet such parameters are beyond the scope of the present approach based on the leading orders. Hence further investigations of this issues are called for.

\section{SUMMARY}

We applied two methods to study the phase diagram and the magnetic susceptibility of the isotropic antiferromagnetic spin 1/2 Heisenberg model coupled to Einstein phonons via a bond coupling: the flow equation method and a quantum Monte Carlo approach.

The flow equation approach maps the full problem onto an effective magnetic problem, i. e. onto a frustrated spin chain. The calculation was performed in leading order in $g / \omega$ and in the two leading orders in $J / \omega$ and is thus systematic to order $g^{2} / \omega^{2}$ and $g^{2} J / \omega^{3}$. The expansion approach works especially well in the antiadiabatic limit.

To apply the flow equation formalism, it is important to use the spin operator which couples to the phonons in its normal-ordered form. To obtain $\chi(T)$ from the calculated effective couplings $J_{1}(T)$ and $J_{2}(T)$ we used high temperature series expansions and exact complete diagonalization.

The quantum Monte Carlo calculations are completely independent of the effective couplings and are based on a loop algorithm. Spin and phonon degrees of freedom are dealt with an equal footing.

The zero temperature phase diagram derived by these methods agrees well for intermediate $J / \omega$ and is in agreement with the result in Ref. 14. Approaching the limit $J / \omega \rightarrow 0$ we find a finite critical spin-phonon coupling $g_{\mathrm{c}} / \omega$ as an important difference to the difference coupling. This is rigorous since a vanishing $g_{\mathrm{c}} / \omega$ for $J / \omega$ would correspond to the limit where our approach becomes exact. We cannot fix the exact value of $g_{\mathrm{c}} / \omega$ in this limit as the flow equation approach corresponds to a leading order expansion in $J / \omega$ and $g / \omega$ and thus is not exact for finite $g / \omega$. Yet both our approaches yield a significantly lower value of $g_{\mathrm{c}} / \omega$ than the fourth order calculation 10 .

We compared the magnetic susceptibilities obtained by both methods and find good agreement. We conclude that at least in this parameter regime the thermodynamic properties of the Hamiltonian Eq. 1 can be described excellently by an effective spin model with temperature dependent couplings. The restriction in $g / \omega$ and $J / \omega$ for the the flow equations are due the leading order character of the approach presented here and could be overcome by a more advanced analysis. The flow equation method provides a good completion to an elaborate QMC analysis as it can be used more quickly, so that the fitting of parameters is easier. 


\section{Acknowledgments}

The authors acknowledge helpful discussions with A. Bühler, K. Fabricius, H. Fehske, A. Klümper, E. Müller-
Hartmann, and A. Weiße. RK and CR were supported by the Deutsche Forschungsgemeinschaft (DFG). GSU acknowledges funding by the DFG in the Schwerpunkt 1073 .
* Electronic address: e-mail: cr@thp.uni-koeln.de

$\dagger$ Electronic address: ul@thp.uni-koeln.de

¥ Electronic address: gu@thp.uni-koeln.de

1 J. W. Bray, L. V. Interrante, I. S. Jacobs, and J. C. Bonner, in Extended Linear Chain Compounds, edited by J. S. Miller (Plenum Press, New York, 1983), vol. 3, p. 353.

2 E. Pytte, Phys. Rev. B 10, 4637 (1974).

3 M. C. Cross and D. S. Fisher, Phys. Rev. B 19, 402 (1979).

${ }^{4}$ M. Hase, I. Terasaki, and K. Uchinokura, Phys. Rev. Lett. 70, 3651 (1993).

5 J. P. Boucher and L. P. Regnault, J. Phys. I France 6, 1939 (1996).

6 R. J. Bursill, R. H. McKenzie, and C. J. Hamer, Phys. Rev. Lett. 83, 408 (1999).

7 P. Sun, D. Schmeltzer, and A. R. Bishop, Phys. Rev. B 62, 11308 (2000).

8 S. Trebst, N. Elstner, and H. Monien, cond-mat/9907266 (1999).

9 G. Wellein, H. Fehske, and A. P. Kampf, Phys. Rev. Lett. 81, 3956 (1998).

10 A. Weiße, G. Wellein, and H. Fehske, Phys. Rev. B 60, 6566 (1999).

11 G. S. Uhrig, Phys. Rev. B 57, 14004 (1998).

12 C. Raas, A. Bühler, and G. S. Uhrig, Eur. Phys. J. B 21, 369 (2001).

13 A. W. Sandvik, R. R. P. Singh, and D. K. Campbell, Phys. Rev. B 56, 14510 (1997).

14 A. W. Sandvik and D. K. Campbell, Phys. Rev. Lett. 83, 195 (1999).

15 R. W. Kühne and U. Löw, Phys. Rev. B 60, 12125 (1999).

16 I. Affleck, D. Gepner, H. J. Schulz, and T. Ziman, J. Phys. A: Math. Gen. 22, 511 (1989).

17 K. Okamoto and K. Nomura, Phys. Lett. A169, 433 (1992).

18 S. Eggert, Phys. Rev. B 54, 9612 (1996).

19 F. J. Wegner, Ann. Physik 3, 77 (1994).
20 F. J. Wegner, Phys. Rep. 348, 77 (2001).

${ }^{21}$ H. Fröhlich, Phys. Roy. Soc. Lond. A215, 291 (1952).

22 S. K. Kehrein, A. Mielke, and P. Neu, Z. Phys. B 99, 269 (1996)

23 A. Mielke, Eur. Phys. J. B 5, 605 (1998).

24 C. Knetter and G. S. Uhrig, Eur. Phys. J. B 13, 209 (2000).

25 H. Fehske, M. Holicki, and A. Weiße, in Advances in Solid State Physics, edited by B. Kramer (Springer, Berlin, 2000), vol. 40, p. 235.

26 W. Geertsma and D. Khomskii, Phys. Rev. B 54, 3011 (1996).

27 D. Khomskii, W. Geertsma, and M. Mostovoy, Czech. Journ. of Physics 46, 3239 (1996).

28 R. Werner, C. Gros, and M. Braden, Phys. Rev. B 59, 14356 (1999).

29 A. Bühler, N. Elstner, and G. S. Uhrig, Eur. Phys. J. B 16, 475 (2000).

30 C. Gros and R. Werner, Phys. Rev. B 58, 14677 (1998).

31 G. S. Uhrig and B. Normand, Phys. Rev. B 63, 134418 (2001).

32 A. Bühler, U. Löw, and G. S. Uhrig, Phys. Rev. B 64, 024428 (2001).

33 A. Klümper, Z. Phys. B 91, 507 (1993).

34 S. Eggert, I. Affleck, and M. Takahashi, Phys. Rev. Lett. 73, 332 (1994).

35 A. Klümper, Eur. Phys. J. B 5, 677 (1998).

${ }^{36}$ H. G. Evertz, G. Lana, and M. Marcu, Phys. Rev. Lett. 70, 875 (1993).

37 G. Castilla, S. Chakravarty, and V. J. Emery, Phys. Rev. Lett. 75, 1823 (1995).

38 J. Riera and A. Dobry, Phys. Rev. B 51, 16098 (1995).

${ }^{39}$ K. Fabricius, A. Klümper, U. Löw, B. Büchner, T. Lorenz, G. Dhalenne, and A. Revcolevschi, Phys. Rev. B 57, 1102 (1998). 